\title{
A New Parametric Differential Operator of p-Valently Analytic Functions
}

\author{
Samir B. Hadid, ${ }^{1,2}$ Rabha W. Ibrahim $\left(\mathbb{D},{ }^{3}\right.$ and G. Murugusundaramoorthy ${ }^{4}{ }^{4}$ \\ ${ }^{1}$ Nonlinear Dynamics Research Center (NDRC), Ajman University, Ajman, UAE \\ ${ }^{2}$ Department of Mathematics and Sciences, College of Humanities and Sciences, Ajman University, 346 Ajman, UAE \\ ${ }^{3}$ IEEE: 94086547, Kuala Lumpur 59200, Malaysia \\ ${ }^{4}$ School of Advanced Sciences, VIT University, Vellore 14, India
}

Correspondence should be addressed to Rabha W. Ibrahim; rabhaibrahim@yahoo.com

Received 23 August 2021; Revised 12 October 2021; Accepted 16 October 2021; Published 31 October 2021

Academic Editor: Mohsan Raza

Copyright (c) 2021 Samir B. Hadid et al. This is an open access article distributed under the Creative Commons Attribution License, which permits unrestricted use, distribution, and reproduction in any medium, provided the original work is properly cited.

\begin{abstract}
Newly, numerous investigations are considered utilizing the idea of parametric operators (integral and differential). The objective of this effort is to formulate a new 2D-parameter differential operator (PDO) of a class of multivalent functions in the open unit disk. Consequently, we formulate the suggested operator in some interesting classes of analytic functions to study its geometric properties. The recognized class contains some recent works.
\end{abstract}

\section{Introduction}

In analysis, a parametric differential operator (PDO) is a differential operator of a dependent variable with respect to another dependent variable that is engaged when both variables formulate on an independent third variable, typically supposed as "time." We shall use this idea to consider the PDO of a complex variable to discuss its properties in the opinion of the geometric function theory (GFT). The field of differential operators is investigated in GFT early by the well-known Salagean differential operator and the Ruscheweyh derivative. Later, these operators are generalized by different types of parameters using a $1 \mathrm{D}$-parameter fractional differential operator [1] and 2D-parameter fractional differential operator [2]. Recently, using the class of normalized functions $\psi \in \Sigma$

$$
\psi(\zeta)=\zeta+\sum_{n=2}^{\infty} \psi_{n} \zeta^{n}, \zeta \in \Delta:=\{\zeta \in \mathbb{C}:|\zeta|<1\}
$$

Ibrahim and Jay [3] presented PDO of the following form: for $\alpha \in[0,1]$

$$
\begin{aligned}
& \mathscr{P}^{0} \psi(\zeta)=\psi(\zeta), \\
& \mathscr{P}^{\alpha} \psi(\zeta)=\frac{\rho_{1}(\alpha, \zeta)}{\rho_{1}(\alpha, \zeta)+\rho_{0}(\alpha, \zeta)} \psi(\zeta)+\frac{\rho_{0}(\alpha, \zeta)}{\rho_{1}(\alpha, \zeta)+\rho_{0}(\alpha, \zeta)}\left(\zeta \psi^{\prime}(\zeta)\right) .
\end{aligned}
$$

The functions $\rho_{1}, \rho_{0}:[0,1] \times \Delta \longrightarrow \Delta$ are analytic in $\Delta$ satisfying $\rho_{1}(\alpha, \zeta) \neq-\rho_{0}(\alpha, \zeta)$.

$$
\lim _{\alpha \longrightarrow 0} \rho_{1}(\alpha, \zeta)=1, \lim _{\alpha \longrightarrow 1} \rho_{1}(\alpha, \zeta)=0, \rho_{1}(\alpha, \zeta) \neq 0, \forall \zeta \in \Delta, \alpha \in(0,1),
$$

$$
\lim _{\alpha \longrightarrow 0} \rho_{0}(\alpha, \zeta)=0, \lim _{\alpha \longrightarrow 1} \rho_{0}(\alpha, \zeta)=1, \rho_{0}(\alpha, \zeta) \neq 0, \forall \zeta \in \Delta, \alpha \in(0,1)
$$


More studies are given by Ibrahim and Baleanu $[4,5]$ using (2) to present a hybrid diff-integral operator and a quantum hybrid operator, respectively.

In this effort, we generalize (2) by considering another class of analytic functions denoting by $\Sigma_{\wp}$ and constructing by

$$
\psi(\zeta)=\zeta^{\wp}+\sum_{n=\wp+1}^{\infty} \psi_{n} \zeta^{n}, \wp \in \mathbb{N}
$$

which are analytic in $\Delta$. Recently, different investigations are presented studying the geometric behavior of this class (see [6-9]).

The Hadamard product $[10,11]$ for two functions in $\Sigma_{\wp}$ is given by the series

$$
\begin{aligned}
(\psi * \varphi)(\zeta) & =\left(\zeta^{\wp}+\sum_{n=\wp+1}^{\infty} \psi_{n} \zeta^{n}\right) *\left(\zeta^{\wp}+\sum_{n=\wp+1}^{\infty} \varphi_{n} \zeta^{n}\right) \\
& =\zeta^{\wp}+\sum_{n=\wp+1}^{\infty}\left(\psi_{n} \varphi_{n}\right) \zeta^{n} \in \Sigma_{\wp} .
\end{aligned}
$$

Definition 1. For a function $\psi \in \Sigma_{\wp}$, PDO is defined as follows:

$$
\begin{aligned}
& \mathbb{Q}^{0} \psi(\zeta)=\psi(\zeta) \\
& \mathscr{Q}^{\alpha} \psi(\zeta)=\left(\frac{\rho_{1}(\alpha, \zeta)}{\rho_{1}(\alpha, \zeta)+\rho_{0}(\alpha, \zeta)}\right) \psi(\zeta)+\left(\frac{\rho_{0}(\alpha, \zeta)}{\rho_{1}(\alpha, \zeta)+\rho_{0}(\alpha, \zeta)}\right)\left(\frac{\zeta}{\wp}\right) \psi^{\prime}(\zeta) \\
&=\zeta^{\wp}+\sum_{n=\wp+1}^{\infty} \psi_{n}\left(\frac{\rho_{1}(\alpha, \zeta)+(n / \wp) \rho_{0}(\alpha, \zeta)}{\rho_{1}(\alpha, \zeta)+\rho_{0}(\alpha, \zeta)}\right) \zeta^{n}, \\
& \mathbb{Q}^{2 \alpha} \psi(\zeta)=\mathbb{Q}\left(\mathbb{Q}^{\alpha} \psi(\zeta)\right)=\zeta^{\wp}+\sum_{n=\wp+1}^{\infty} \psi_{n}\left(\frac{\rho_{1}(\alpha, \zeta)+(n / \wp) \rho_{0}(\alpha, \zeta)}{\rho_{1}(\alpha, \zeta)+\rho_{0}(\alpha, \zeta)}\right)^{2} \zeta^{n}, \vdots \\
& \mathbb{Q}^{m \alpha} \psi(\zeta)=\mathbb{Q}^{\alpha}\left[\mathcal{Q}^{(m-1) \alpha} \psi(\zeta)\right]=\zeta^{\wp}+\sum_{n=\wp+1}^{\infty} \psi_{n}\left(\frac{\rho_{1}(\alpha, \zeta)+(n / \wp) \rho_{0}(\alpha, \zeta)}{\rho_{1}(\alpha, \zeta)+\rho_{0}(\alpha, \zeta)}\right)^{m} \zeta^{n}, \\
& \mathbb{Q}^{m \alpha} \psi(\zeta)=\zeta^{\wp}+\sum_{n=\wp+1}^{\infty} \psi_{n} \Lambda_{n}^{m} \zeta^{n}, \\
&(\zeta \in \Delta, \wp \in \mathbb{N}, \alpha \in[0,1], m \in \mathbb{N}),
\end{aligned}
$$

where

$$
\Lambda_{n}=\frac{\rho_{1}(\alpha, \zeta)+(n / \wp) \rho_{0}(\alpha, \zeta)}{\rho_{1}(\alpha, \zeta)+\rho_{0}(\alpha, \zeta)}
$$

$\rho_{1}$ and $\rho_{0}$ are defined in (3) and (4), respectively.

\section{Remark 2.}

(i) It is clear that $Q^{m \alpha} \psi(\zeta) \in \Sigma_{\wp}$, and it is a generalization of $(2)(\wp=1)$

(ii) The integral operator that corresponds to $Q^{m \alpha} \psi(\zeta)$ is

$$
\mathscr{L}^{m \alpha} \psi(\zeta)=\zeta^{\wp}+\sum_{n=\wp+1}^{\infty} \frac{\psi_{n}}{\Lambda_{n}^{m}} \zeta^{n} \cdot(\zeta \in \Delta, \wp \in \mathbb{N}, \alpha \in[0,1]),
$$

where

$$
\left(\mathbb{Q}^{m \alpha} * \mathscr{L}^{m \alpha}\right) \psi(\zeta)=\left(\mathscr{L}^{m \alpha} * \mathbb{Q}^{m \alpha}\right) \psi(\zeta)=\psi(\zeta)
$$

Moreover, we have the following property:

Proposition 3 (semigroup property). Consider the PDO; then for $\psi$ and $\varphi \in \Sigma_{\wp}$

$$
\mathbb{Q}^{m \alpha}[a \psi(\zeta)+b \varphi(\zeta)]=a \mathbb{Q}^{m \alpha} \psi(\zeta)+b \mathbb{Q}^{m \alpha} \varphi(\zeta), a, b \in \mathbb{R}
$$

Proof. Let $m=1$; the definition of $\mathbb{Q}^{m v}$ implies

$$
\begin{aligned}
\mathcal{Q}^{\alpha}[a \psi(\zeta)+b \varphi(\zeta)]= & \left(\frac{\rho_{1}(\alpha, \zeta)}{\rho_{1}(\alpha, \zeta)+\rho_{0}(\alpha, \zeta)}\right)[a \psi(\zeta)+b \varphi(\zeta)] \\
& +\left(\frac{\rho_{0}(\alpha, \zeta)}{\rho_{1}(\alpha, \zeta)+\rho_{0}(\alpha, \zeta)}\right)\left(\frac{\zeta}{\wp}\right)[a \psi(\zeta)+b \varphi(\zeta)]^{\prime} \\
= & a\left(\left(\frac{\rho_{1}(\alpha, \zeta)}{\rho_{1}(\alpha, \zeta)+\rho_{0}(\alpha, \zeta)}\right) \psi(\zeta)\right. \\
& \left.+\left(\frac{\rho_{0}(\alpha, \zeta)}{\rho_{1}(\alpha, \zeta)+\rho_{0}(\alpha, \zeta)}\right)\left(\frac{\zeta}{\wp}\right) \psi^{\prime}(\zeta)\right) \\
& +b\left(\left(\frac{\rho_{1}(\alpha, \zeta)}{\rho_{1}(\alpha, \zeta)+\rho_{0}(\alpha, \zeta)}\right) \varphi(\zeta)\right. \\
& \left.+\left(\frac{\rho_{0}(\alpha, \zeta)}{\rho_{1}(\alpha, \zeta)+\rho_{0}(\alpha, \zeta)}\right)\left(\frac{\zeta}{\wp}\right) \varphi^{\prime}(\zeta)\right) \\
= & a Q^{\alpha} \psi(\zeta)+b Q^{\alpha} \varphi(\zeta)
\end{aligned}
$$

Hence, for all $m$, we have the desired assertion.

Our study is about the following class:

Definition 4. A function $\psi \in \Sigma_{\wp}$ is called in the class $\Sigma_{\wp}^{\alpha}(\sigma, p)$ if it satisfies the inequality

$$
\frac{(1-\sigma)}{\zeta^{\wp}}\left[Q^{m \alpha} \psi(\zeta)\right]+\left(\frac{\sigma}{\wp \zeta^{\wp-1}}\right)\left[Q^{m \alpha} \psi(\zeta)\right]^{\prime} \prec p(\zeta)=\frac{\mu \zeta+1}{\nu \zeta+1},
$$

$$
(\zeta \in \Delta, \alpha, \sigma \in[0,1],-1 \leq \nu<\mu \leq 1, \wp \in \mathbb{N}),
$$

where the symbol $\prec$ presents the subordination symbol [12] and $p$ is convex univalent in $\Delta$.

For example

$$
p(\zeta)=\frac{\mu \zeta+1}{\nu \zeta+1}=Y_{\mu, v}(\zeta)
$$

which is univalent convex in $\Delta$, and it is the extreme function in the set

$$
\mathscr{P}:=\left\{p \in \Delta: p(\zeta)=1+\sum_{n=1}^{\infty} p_{i} \zeta^{n}\right\} .
$$


Define a functional $\Psi: \Delta \longrightarrow \Delta$, as follows:

$$
\begin{aligned}
\Psi(\zeta) & :=\frac{(1-\sigma)}{\zeta^{\wp}}\left[\mathcal{Q}^{m \alpha} \psi(\zeta)\right]+\left(\frac{\sigma}{\wp \zeta^{\wp-1}}\right)\left[\mathcal{Q}^{m \alpha} \psi(\zeta)\right]^{\prime}, \\
& =1+\sum_{n=\wp+1}^{\infty} \Psi_{n+\wp} \psi_{n} \zeta^{n-\wp}, \zeta \in \Delta,
\end{aligned}
$$

where

$$
\Psi_{n+\wp}=\left(1+\frac{n-1}{\wp} \sigma\right) \Lambda_{n}^{m} .
$$

Shortly, by Definition 4 we say

$$
\Psi(\zeta) \prec Y_{\mu, v}(\zeta):=\frac{\mu \zeta+1}{v \zeta+1}, \zeta \in \Delta .
$$

Our aim is to study the operator formula $\Psi$. We recall the following results:

Lemma 5 (see [12]). Let two analytic functions $f(\zeta)$ and $g(\zeta)$ be convex univalent defined in $\Delta$ such that $f(0)=g(0)$. Moreover, for a constant $c \neq 0, \mathfrak{R}(c) \geq 0$, the subordination

$$
f(\zeta)+(1 / c) f^{\prime}(\zeta) \prec g(\zeta)
$$

implies

$$
f(\zeta) \prec g(\zeta)
$$

Lemma 6 (see [12]). Define the general class of holomorphic functions

$$
\mathbb{H}[a, n]=\left\{h: h(\zeta)=a+a_{n} \zeta^{n}+a_{n+1} \zeta^{n+1}+\cdots\right\},
$$

where $a \in \mathbb{C}$ and $n$ is a positive integer. If $c \in \mathbb{R}$, then

$$
\mathfrak{R}\left\{h(\zeta)+c \zeta h^{\prime}(\zeta)\right\}>0 \Rightarrow \mathfrak{R}(h(\zeta))>0 .
$$

Moreover, if $c>0$ and $h \in \mathbb{H}[1, n]$, then there are fixed numbers $\ell_{1}>0$ and $\ell_{2}>0$ with the inequality

$$
\begin{aligned}
& h(\zeta)+c \zeta h^{\prime}(\zeta) \prec\left(\frac{1+\zeta}{1-\zeta}\right)^{\ell_{1}} \\
& h(\zeta) \prec\left(\frac{1+\zeta}{1-\zeta}\right)^{\ell_{2}} .
\end{aligned}
$$

Lemma 7 (see [13]). Let $\hbar, p \in \mathbb{H}[a, n]$, where $p$ is convex univalent in $\Delta$ and for $\mathbb{k}_{1}, \mathbb{k}_{2} \in \mathbb{C}, \mathbb{k}_{2} \neq 0$; then

$$
\mathbb{k}_{1} \hbar(\zeta)+\mathbb{k}_{2} \zeta \hbar^{\prime}(\zeta)<\mathbb{k}_{1} p(\zeta)+\mathbb{k}_{2} \zeta p^{\prime}(\zeta) \longrightarrow \hbar(\zeta) \prec p(\zeta)
$$

Lemma 8 (see [14]). Let $h, p \in \mathbb{H}[a, n]$, where $p$ is convex univalent in $\Delta$ such that $h(\zeta)+\mathbb{k} \zeta h^{\prime}(\zeta)$ is univalent; then

$$
p(\zeta)+\mathbb{k} \zeta p^{\prime}(\zeta) \prec h(\zeta)+\mathbb{k} \zeta h^{\prime}(\zeta) \longrightarrow p(\zeta) \prec h(\zeta) .
$$

Lemma 9 (see [15]). Let $\hbar, y, g \in \mathbb{H}[a, n]$, and $g$ is convex univalent in $\Delta$ such that $\hbar<g$ and $y<g$; then

$$
\mathbb{k} \hbar+(1-\mathbb{k}) y<g, \mathbb{k} \in[0,1] .
$$

\section{The Results}

In this section, we illustrate our main results concerning the class $\Sigma_{\mathfrak{q}}^{\alpha}(\sigma, p)$ for some special $p(\zeta), \zeta \in \Delta$.

\subsection{General Properties}

Theorem 10. Suppose that $\psi \in \Sigma_{\mathfrak{\wp}}^{\alpha}(\sigma, p)$. If $\mathfrak{R}\{\Psi(\zeta)\}>0$, then the coefficient bounds of $\Psi$ satisfy the inequality

$$
\frac{\left|\Psi_{n}\right|}{2} \leq \int_{0}^{2 \pi}\left|e^{-i n \theta}\right| d \mathfrak{M}(\theta)
$$

where $d \mathfrak{M}$ is a probability measure. Also, if

$$
\mathfrak{R}\left(e^{i \chi} \Psi(\zeta)\right)>0, \zeta \in \Delta, \chi \in \mathbb{R},
$$

then $\psi \in \sum_{\mathfrak{\wp}}^{\alpha}(\sigma,(\nu \zeta+1) /(v \zeta+1))$, that is

$$
\Psi(\zeta) \approx \frac{\mu \zeta+1}{\nu \zeta+1}, \zeta \in \Delta
$$

Proof. By the assumption, we have

$$
\mathfrak{R}(\Psi(\zeta))=\mathfrak{R}\left(1+\sum_{n=\mathfrak{\wp}+1}^{\infty} \Psi_{n} \zeta^{n}\right)>0 .
$$

Thus, the Carathéodory positivist technique yields

$$
\left|\psi_{n}\right| \leq 2 \int_{0}^{2 \pi}\left|e^{-i n \theta}\right| d \mathfrak{M}(\theta)
$$

where $d \mathfrak{M}$ is a probability measure. In addition, if

$$
\mathfrak{R}\left(e^{i \chi} \Psi(\zeta)\right)>0, \zeta \in \Delta, \chi \in \mathbb{R},
$$

then according to Theorem 1.6 in [10] and for fixed $\chi \in \mathbb{R}$, we have

$$
\Psi(\zeta) \approx p(\zeta)=\frac{\mu \zeta+1}{\nu \zeta+1}, \zeta \in \Delta
$$

Hence, $\psi \in \sum_{\wp}^{\alpha}(\sigma,(\nu \zeta+1) /(\nu \zeta+1))$.

The next results show the sufficient and necessary conditions for the sandwich behavior of the functional $\Psi(\zeta)=\left(1-\sigma / \zeta^{\wp}\right)\left[Q^{m \alpha} \psi(\zeta)\right]+\left(\sigma / \wp \zeta^{\wp-1}\right)\left[\mathbb{Q}^{m \alpha} \psi(\zeta)\right]^{\prime}$. 
Theorem 11. Let the following assumptions hold

$$
\frac{\sigma \zeta\left[Q^{m \alpha} \psi(\zeta)\right]^{\prime \prime}+(-2 \sigma \wp+2 \sigma+\wp)\left[Q^{m \alpha} \psi(\zeta)\right]^{\prime}+(\sigma-1)(\wp-1) \wp\left[Q^{m \alpha} \psi(\zeta)\right]}{\wp \zeta^{\wp-1}} \prec p_{2}(\zeta)+\zeta p_{2}^{\prime}(\zeta)
$$

where $p_{2}(0)=1$ and convex in $\Delta$. Moreover, let $\Psi(\zeta)$ be univalent in $\Delta$ such that $\Psi \in \mathbb{H}\left[p_{1}(0), 1\right] \cap \mathbb{Q}$, where $\mathbb{Q}$ repre- sents the set of all injection analytic functions $f$ with $\lim _{\zeta \in \partial \Delta} f \neq \infty$ and

$$
p_{1}(\zeta)+\zeta p_{1}{ }^{\prime}(\zeta) \prec \frac{\left.\sigma \zeta\left[Q^{m \alpha} \psi(\zeta)\right]^{\prime \prime}+(-2 \sigma \wp+2 \sigma+\wp)\left[Q^{m \alpha} \psi(\zeta)\right]^{\prime}\right)+(\sigma-1)(\wp-1) \wp\left[Q^{m \alpha} \psi(\zeta)\right]}{\wp \zeta^{\wp-1}} .
$$

Then

$$
p_{1}(\zeta) \prec \Psi(\zeta) \prec p_{2}(\zeta)
$$

and $p_{1}(\zeta)$ is the best subdominant, and $p_{2}(\zeta)$ is the best dominant.

Proof. Since

$$
\Psi(\zeta)+\zeta \Psi^{\prime}(\zeta)=\frac{\left.\sigma \zeta\left[Q^{m \alpha} \psi(\zeta)\right]^{\prime \prime}+(-2 \sigma \wp+2 \sigma+\wp)\left[Q^{m \alpha} \psi(\zeta)\right]^{\prime}\right)+(\sigma-1)(\wp-1) \wp\left[Q^{m \alpha} \psi(\zeta)\right]}{\wp \zeta^{\wp-1}}
$$

then we obtain the next double inequality

$$
p_{1}(\zeta)+\zeta p_{1}^{\prime}(\zeta) \prec \Psi(\zeta)+\zeta \Psi^{\prime}(\zeta) \prec p_{2}(\zeta)+\zeta p_{2}^{\prime}(\zeta)
$$

Thus, Lemmas 7 and 8 imply the desired assertion.

Theorem 12. Let $p$ be a univalent convex function in $\Delta$ such that $p(0)=0$ and

$$
\left[Q^{m \alpha} \psi(\zeta)\right] \prec p(\zeta),\left[\mathscr{L}^{m \alpha} \psi(\zeta)\right] \prec p(\zeta)
$$

Then

Proof. By the definition of $\left[\mathscr{Q}^{m \alpha} \psi(\zeta)\right]$ and $\left[\mathscr{L}^{m \alpha} \psi(\zeta)\right]$, clearly we have $\left[\mathscr{A}^{m \alpha} \psi(\zeta)\right] \in \Sigma_{\wp}$. Hence, a direct application of Lemma 9, we obtain the result.

2.2. Inclusion Properties. In this part, we deal with the inclusion properties.

Theorem 13. For $\sigma_{2} \leq \sigma_{1}<0$ and $\psi \in \Sigma_{\wp}$, then

$$
\sum_{\wp}^{\alpha}\left(\sigma_{2}, p\right) \subset \sum_{\wp}^{\alpha}\left(\sigma_{1}, p\right)
$$

Proof. Let $\psi \in \Sigma_{\wp}^{\alpha}\left(\sigma_{2}, p\right)$. Define the analytic function in $\Delta$, as follows:

$$
\phi(\zeta)=\zeta^{-\wp}\left[Q^{m \alpha} \psi(\zeta)\right]
$$

satisfying $\phi(0)=1$. A computation gives

$$
\frac{\left(1-\sigma_{2}\right)}{\zeta^{\wp}}\left[Q^{m \alpha} \psi(\zeta)\right]+\left(\frac{\sigma_{2}}{\wp \zeta^{\wp-1}}\right)\left[Q^{m \alpha} \psi(\zeta)\right]^{\prime}=\phi(\zeta)+\frac{\sigma_{2}}{\wp}\left(\zeta \phi^{\prime}(\zeta)\right) \text {. }
$$

Consequently, we get the inequality

$$
\phi(\zeta)+\frac{\sigma_{2}}{\wp}\left(\zeta \phi^{\prime}(\zeta)\right) \prec \frac{\mu \zeta+1}{v \zeta+1}
$$

Applying Lemma 5 with $\sigma_{2} / \wp>0$ gives

$$
\phi(\zeta) \prec \frac{\mu \zeta+1}{\nu \zeta+1}
$$


Since $0<\sigma_{1} / \sigma_{2}<1$ and $Y_{\mu, v}(\zeta)$ is convex univalent in $\Delta$, we arrive at the inequality

$$
\begin{aligned}
\frac{\left(1-\sigma_{1}\right)}{\zeta^{\wp}}\left[Q^{m \alpha} \psi(\zeta)\right]+\left(\frac{\sigma_{1}}{\wp \zeta^{\wp-1}}\right)\left[Q^{m \alpha} \psi(\zeta)\right]^{\prime} \\
=\left(1-\sigma_{1}\right) \phi(\zeta)+\left(\frac{\sigma_{1}}{\wp \zeta^{\wp-1}}\right)\left[Q^{m \alpha} \psi(\zeta)\right]^{\prime} \\
=\left(1-\sigma_{1}\right) \phi(\zeta)+\frac{\sigma_{1}}{\wp}\left(\zeta \phi^{\prime}(\zeta)+\wp \phi(\zeta)\right), \\
=\left(1-\sigma_{1}\right) \phi(\zeta)+\frac{\sigma_{1}}{\wp}\left(\zeta \phi^{\prime}(\zeta)+\wp \phi(\zeta)\right)+\left(\frac{\sigma_{1}}{\sigma_{2}} \phi(\zeta)-\frac{\sigma_{1}}{\sigma_{2}} \phi(\zeta)\right) \\
=\frac{\sigma_{1}}{\sigma_{2}}\left(1-\sigma_{2}\right) \phi(\zeta)+\frac{\sigma_{2}}{\wp}\left(\zeta \phi^{\prime}(\zeta)+\wp \phi(\zeta)\right)+\left(1-\frac{\sigma_{1}}{\sigma_{2}}\right) \phi(\zeta) \\
=\frac{\sigma_{1}}{\sigma_{2}}\left[\frac{\left(1-\sigma_{2}\right)}{\zeta^{\wp}}\left[Q^{m \alpha} \psi(\zeta)\right]+\frac{\sigma_{2}}{\wp \zeta^{\wp-1}}\left[Q^{m \alpha} \psi(\zeta)\right]^{\prime}\right] \\
\quad+\left(1-\frac{\sigma_{1}}{\sigma_{2}}\right) \phi(\zeta) \prec \frac{\mu \zeta+1}{v \zeta+1}=Y_{\mu, \nu}(\zeta) .
\end{aligned}
$$

Hence, by Definition 4 , we conclude that $\psi \in \Sigma_{\mathfrak{\wp}}^{\alpha}\left(\sigma_{1}, p\right)$.

Theorem 14. Let

$$
\Psi(\zeta)=\frac{(1-\sigma)}{\zeta^{\wp}}\left[Q^{m \alpha} \psi(\zeta)\right]+\left(\frac{\sigma}{\wp \zeta^{\wp-1}}\right)\left[Q^{m \alpha} \psi(\zeta)\right]^{\prime} .
$$

Then

$$
\begin{aligned}
& \frac{\left[Q^{m \alpha} \psi(\zeta)\right]^{\prime}}{\zeta^{\wp}} \hbar_{1}+\frac{\left[Q^{m \alpha} \psi(\zeta)\right]}{\zeta^{\wp-1}}\left[\hbar_{1}+(1+\wp) \hbar_{2}+\hbar_{2}\right] \\
& +\hbar_{2} \zeta^{2-\wp}\left[Q^{m \alpha} \psi(\zeta)\right]^{\prime \prime}<\left(\frac{1+\zeta}{1-\zeta}\right)^{\ell_{1}} \Rightarrow \Psi(\zeta)<\left(\frac{1+\zeta}{1-\zeta}\right)^{\ell_{2}}
\end{aligned}
$$

where

$$
\begin{aligned}
\ell_{1} & >0, \ell_{2}>0, \\
\hbar_{1} & =1-\sigma, \\
\hbar_{2} & =\frac{\sigma}{\wp}, \\
\wp & >0 .
\end{aligned}
$$

Proof. A calculation implies that

$$
\begin{aligned}
\Psi(\zeta)+\zeta \Psi^{\prime}(\zeta)= & \frac{(1-\sigma)}{\zeta^{\wp}}\left[Q^{m \alpha} \psi(\zeta)\right]+\left(\frac{\sigma}{\wp \zeta^{\wp-1}}\right)\left[Q^{m \alpha} \psi(\zeta)\right]^{\prime} \\
& +\zeta\left(\frac{(1-\sigma)}{\zeta^{\wp}}\left[Q^{m \alpha} \psi(\zeta)\right]+\left(\frac{\sigma}{\wp \zeta^{\wp-1}}\right)\left[Q^{m \alpha} \psi(\zeta)\right]^{\prime}\right) \\
= & \frac{\left[Q^{m \alpha} \psi(\zeta)\right]^{\prime}}{\zeta^{\wp}} \hbar_{1}+\frac{\left[Q^{m \alpha} \psi(\zeta)\right]}{\zeta^{\wp-1}}\left[\hbar_{1}+(1+\wp) \hbar_{2}+\hbar_{2}\right] \\
& +\hbar_{2} \zeta^{2-\wp}\left[Q^{m \alpha} \psi(\zeta)\right]^{\prime \prime}<\left(\frac{1+\zeta}{1-\zeta}\right)^{\ell_{1}}
\end{aligned}
$$

According to Lemma 6 joining the value $c=1$, we get

$$
\Psi(\zeta)<\left(\frac{1+\zeta}{1-\zeta}\right)^{\ell_{2}}
$$

Corollary 15. Let $\Psi(\zeta)$ be assumed as in Theorem14. If the subordination

$$
\begin{aligned}
& \frac{\left[Q^{m \alpha} \psi(\zeta)\right]^{\prime}}{\zeta^{\wp}} \hbar_{1}+\frac{\left[Q^{m \alpha} \psi(\zeta)\right]}{\zeta^{\wp-1}}\left[\hbar_{1}+(1+\wp) \hbar_{2}+\hbar_{2}\right] \\
& +\hbar_{2} \zeta^{2-\wp}\left[Q^{m \alpha} \psi(\zeta)\right]^{\prime \prime}<\left(\frac{1+\zeta}{1-\zeta}\right),
\end{aligned}
$$

where $\ell_{1}>0, \ell_{2}>0, \hbar_{1}=1-\sigma, \hbar_{2}=\sigma / \wp, \wp>0$ holds, then $\psi \in \Sigma_{\wp}^{\alpha}(\sigma,(1+\zeta) /(1-\zeta))$.

Proof. Taking, $\ell_{1}=\ell_{2}=1$ in Theorem 14 implies that $\Psi(\zeta)<(1+\zeta) /(1-\zeta)$. Consequently, we have $\psi \in \Sigma_{\mathfrak{\wp}}^{\alpha}(\sigma$, $(1+\zeta) /(1-\zeta))$

Theorem 16. Let $\psi \in \Sigma_{\wp}^{\alpha}(\sigma, p)$ and $f \in \Sigma_{\wp}$. If

$$
\mathfrak{R}\left(\frac{\mathbb{Q}^{m \alpha} \psi(\zeta)}{\zeta^{\natural}}\right)>\frac{1}{2},
$$

then $\psi \times f \in \Sigma_{\wp}^{\alpha}(\sigma, p)$. 
Proof. A convolution product indicates that

$$
\begin{aligned}
& \frac{(1-\sigma)}{\zeta^{\wp}}\left[Q^{m \alpha}(\psi(\zeta) \times f(\zeta))\right]+\left(\frac{\sigma}{\wp^{\wp-1}}\right)\left[Q^{m \alpha}(\psi(\zeta) \times f(\zeta))\right]^{\prime} \\
& \left.=(1-\sigma)\left[\frac{Q^{m \alpha}(\psi(\zeta)}{\zeta^{\wp}} \times \frac{Q^{m \alpha} f(\zeta)}{\zeta^{\wp}}\right)\right] \\
& \quad+\frac{\sigma}{\wp}\left(\frac{\left.\left[Q^{m \alpha} f(\zeta)\right)\right]^{\prime}}{\zeta^{\wp-1}} \times \frac{Q^{m \alpha} f(\zeta)}{\zeta^{\wp}}\right) \\
& \left.=\left[(1-\sigma)\left[\frac{\left[Q^{m \alpha}(\psi(\zeta)\right.}{\zeta^{\wp}}\right]+\frac{\sigma}{\wp^{\wp-1}}\left[Q^{m \alpha} f(\zeta)\right)\right]^{\prime}\right] \times \frac{Q^{m \alpha} f(\zeta)}{\zeta^{\wp}} \\
& =\Psi(\zeta) \times \frac{Q^{m \alpha} f(\zeta)}{\zeta^{\wp}},
\end{aligned}
$$

where $\Psi(\zeta) \prec Y_{\mu, v}(\zeta)$. In view of real inequality (55), we get that $\left(Q^{m \alpha} f(\zeta) / \zeta^{\wp}\right)$ has the Herglotz integral formula [11].

$$
\frac{Q^{m \alpha} f(\zeta)}{\zeta^{\wp}}=\int_{|\tau|=1} \frac{d \varsigma(\tau)}{1-\tau \zeta},
$$

where $d \varsigma$ conforms the probability measure on the unit circle $|\tau|=1$ and

$$
\int_{|\tau|=1} d \varsigma(\tau)=1
$$

But, $Y_{\mu, v}(\zeta)$ is convex in $\Delta$; then we have

$$
\begin{aligned}
& \frac{(1-\sigma)}{\zeta^{\wp}}\left[Q^{m \alpha}(\psi(\zeta) * f(\zeta))\right]+\left(\frac{\sigma}{\wp \zeta^{\wp-1}}\right)\left[Q^{m \alpha}(\psi(\zeta) * f(\zeta))\right]^{\prime} \\
& \quad=\Theta(\zeta) * \frac{Q^{m \alpha} f(\zeta)}{\zeta^{\wp}} \\
& \quad=\int_{|\tau|=1} \Theta(\tau \zeta) d \varsigma(\tau) \prec Y_{\mu, v}(\zeta) .
\end{aligned}
$$

Thus, $\psi \times f \in \sum_{\wp}^{\alpha}(\sigma, p)$.

2.3. Fekete-Szegö Inequality. In this section, we obtain the Fekete-Szegö relation coefficient estimates for the class $\Sigma_{\wp}^{\alpha}(\sigma, p)$. Let $\Omega$ be the class of functions of the form

$$
\omega(\zeta)=1+\omega_{1} \zeta+\omega_{2} \zeta^{2}+\cdots
$$

in the open unit disk $\Delta$ satisfying $|\omega(z)|<1$. To prove our results, we need the following lemma.

Lemma 17 (see [16]). If $\omega \in \Omega$, then for any complex number $\rho$

$$
\left|\omega_{2}-\rho \omega_{1}^{2}\right| \leq \max \{1,|\rho|\}
$$

The result is sharp for the functions given by $\omega(\zeta)=\zeta$ or $\omega(\zeta)=\zeta^{2}$

Theorem 18. Let the function $\psi$ be formulated by ((5)). Then, $\psi \in \Sigma_{\wp}^{\alpha}(\sigma, p)$ and

$$
\left|\psi_{\wp+2}-\rho \psi_{\wp+1}^{2}\right| \leq\left(\frac{(\mu-v) \wp}{[\wp+(\wp+1) \sigma] \Lambda_{\wp+2}^{m}}\right) \max \{1,|\aleph|\},
$$

where

$$
\aleph=\left(v+\frac{\rho(\mu-v)[\wp+(\wp+1) \sigma] \Lambda_{\wp+2}^{m}}{\wp(1+\sigma)^{2} \Lambda^{2} m_{\wp+1}}\right) .
$$

Proof. Since $\psi \in \Sigma_{\wp}^{\alpha}(\sigma, p)$, we have

$$
\frac{(1-\sigma)}{\zeta^{\wp}}\left[\mathscr{Q}^{m \alpha} \psi(\zeta)\right]+\left(\frac{\sigma}{\wp \zeta^{\wp-1}}\right)\left[\mathscr{Q}^{m \alpha} \psi(\zeta)\right]^{\prime} \prec p(\zeta)=\frac{\mu \zeta+1}{v \zeta+1} .
$$

In addition, there is a Schwarz function $\omega(\zeta)=1+\omega_{1} \zeta+$ $\omega_{2} \zeta^{2}+\cdots$ in $\Omega$ such that

$$
\begin{aligned}
& \frac{(1-\sigma)}{\zeta^{\wp}}\left[Q^{m \alpha} \psi(\zeta)\right]+\left(\frac{\sigma}{\wp \zeta^{\wp-1}}\right)\left[Q^{m \alpha} \psi(\zeta)\right]^{\prime} \prec p(w(\zeta)) p(w(\zeta)) \\
& \quad=\frac{1+\mu w(\zeta)}{1+\nu w(\zeta)} \\
& \quad=1+(\mu-v) \omega_{1} \zeta+\left[(\mu-v) \omega_{2}-v(\mu-v) \omega_{1}^{2}\right] \zeta^{2}+\cdots .
\end{aligned}
$$

Now by (18), we have

$$
\begin{aligned}
& \frac{(1-\sigma)}{\zeta^{\wp}}\left[Q^{m \alpha} \psi(\zeta)\right]+\left(\frac{\sigma}{\wp \zeta^{\wp-1}}\right)\left[Q^{m \alpha} \psi(\zeta)\right]^{\prime} \\
& \quad=1+\sum_{n=\wp+1}^{\infty}\left(1+\frac{n-1}{\wp} \sigma\right) \Lambda_{n}^{m} \psi_{n} \zeta^{n-\wp}, \zeta \in \Delta,
\end{aligned}
$$

where $\Lambda_{n}^{m}$ is given by (19). Equating the coefficients of $\zeta$ and $\zeta^{2}$, we get

$$
(1+\sigma) \Lambda_{\wp+1}^{m} \psi_{\wp+1}=(\mu-v) \omega_{1},
$$

$$
\left(1+\frac{\wp+1}{\wp} \sigma\right) \Lambda_{\wp+2}^{m} \psi_{\wp+2}=(\mu-v) \omega_{2}-v(\mu-v) \omega_{1}^{2},
$$

$$
\left(\frac{\wp+(\wp+1) \sigma}{\wp}\right) \Lambda_{\wp+2}^{m} \psi_{\wp+2}=(\mu-v) \omega_{2}-v(\mu-v) \omega_{1}^{2} .
$$


From (67) and (69), we get

$$
\begin{aligned}
& \psi_{\wp+1}=\frac{(\mu-v) \omega_{1}}{(1+\sigma) \Lambda_{\wp+1}^{m}}, \\
& \psi_{\wp+2}=\frac{(\mu-v) \wp}{[\wp+(\wp+1) \sigma] \Lambda_{\wp+2}^{m}} \omega_{2}-\frac{v(\mu-v) \wp}{[\wp+(\wp+1) \sigma] \Lambda_{\wp+2}^{m}} \omega_{1}^{2} .
\end{aligned}
$$

For any $\rho \in \mathbb{C}$, we get

$$
\begin{aligned}
\psi_{\wp+2}-\rho \psi_{\wp+1}^{2}= & \frac{(\mu-v) \wp}{[\wp+(\wp+1) \sigma] \Lambda_{\wp+2}^{m}} \omega_{2}-\frac{v(\mu-v) \wp}{[\wp+(\wp+1) \sigma] \Lambda_{\wp+2}^{m}} \omega_{1}^{2} \\
& -\left(\frac{(\mu-v) \omega_{1}}{(1+\sigma) \Lambda_{\wp+1}^{m}}\right)^{2}=\frac{(\mu-v) \wp}{[\wp+(\wp+1) \sigma] \Lambda_{\wp+2}^{m}} \\
& \cdot\left[\omega_{2}-\left(\nu+\frac{\rho(\mu-v)[\wp+(\wp+1) \sigma] \Lambda_{\wp+2}^{m}}{\wp(1+\sigma)^{2} \Lambda_{\wp+1}^{2 m}}\right) \omega_{1}^{2}\right] \\
= & \frac{(\mu-v) \wp}{[\wp+(\wp+1) \sigma] \Lambda_{\wp+2}^{m}}\left(\omega_{2}-\aleph \omega_{1}^{2}\right),
\end{aligned}
$$

where

$$
\aleph=\left(v+\frac{\rho(\mu-v)[\wp+(\wp+1) \sigma] \Lambda_{\wp+2}^{m}}{\wp(1+\sigma)^{2} \Lambda 2 m_{\wp+1}}\right) .
$$

By applying Lemma 17, we get

$$
\left|\psi_{\wp+2}-\rho \psi_{\wp+1}^{2}\right| \leq\left(\frac{(\mu-v) \wp}{[\wp+(\wp+1) \sigma] \Lambda_{\wp+2}^{m}}\right) \max \{1,|\aleph|\} .
$$

The result is sharp for the function

$$
\frac{(1-\sigma)}{\zeta^{\wp}}\left[Q^{m \alpha} \psi(\zeta)\right]+\left(\frac{\sigma}{\wp \zeta^{\wp-1}}\right)\left[Q^{m \alpha} \psi(\zeta)\right]^{\prime}=p\left(\zeta^{2}\right)=\frac{\mu \zeta^{2}+1}{v \zeta^{2}+1}
$$

or

$$
\frac{(1-\sigma)}{\zeta^{\wp}}\left[Q^{m \alpha} \psi(\zeta)\right]+\left(\frac{\sigma}{\wp \zeta^{\wp-1}}\right)\left[Q^{m \alpha} \psi(\zeta)\right]^{\prime}=p(\zeta)=\frac{\mu \zeta+1}{v \zeta+1}
$$

Remark 19. By fixing $\rho=1$ in Theorem 18, we get

$$
\left|\psi_{\wp+2}-\psi_{\wp+1}^{2}\right| \leq\left(\frac{(\mu-v) \wp}{[\wp+(\wp+1) \sigma] \Lambda_{\wp+2}^{m}}\right) \max \{1,|\aleph|\},
$$

where

$$
\aleph=\left(v+\frac{(\mu-v)[\wp+(\wp+1) \sigma] \Lambda_{\wp+2}^{m}}{\wp(1+\sigma)^{2} \Lambda 2 m_{\wp+1}}\right) .
$$

From Definition 4, a function $\psi \in \Sigma_{\wp}$ is said to be in the class $\sum_{\wp}^{\alpha}(\sigma, p)$ if it satisfies the inequality (13); then we have

$$
\left|\frac{\Psi(\zeta)-1}{\mu-\nu \Psi(\zeta)}\right|<1
$$

where

$$
\Psi(\zeta)=\frac{(1-\sigma)}{\zeta^{\wp}}\left[Q^{m \alpha} \psi(\zeta)\right]+\left(\frac{\sigma}{\wp \zeta^{\wp-1}}\right)\left[Q^{m \alpha} \psi(\zeta)\right]^{\prime}
$$

is as given in (17).

Now, we obtain coefficient estimates for $f \in \Sigma_{\wp}^{\alpha}(\sigma, p)$.

Theorem 20. Let the function $\psi$ be defined by ((5)). Then, $\psi \in \sum_{\wp}^{\alpha}(\sigma, p)$ if

$$
\begin{aligned}
& \sum_{n=p+1}^{\infty} \Psi_{n, \wp}[1+\nu] \psi_{n} \leq|\mu-\nu|, \\
& \Psi_{n, \wp}=\left(1+\frac{n-1}{\wp} \sigma\right) \Lambda_{n}^{m},
\end{aligned}
$$

where $\Lambda_{n}^{m}$ is given by (8).

Proof. Suppose $\psi$ satisfies (80). Then, for $|\zeta|=r<1$

$$
\begin{aligned}
|\Psi(\zeta)-1|-|\mu-\nu \Psi(\zeta)|= & \left|\sum_{n=p+1}^{\infty} \Psi_{n, \wp} \psi_{n} \zeta^{n-p}\right|-\beta \mid(\mu-v) \\
& +\nu \sum_{n=p+1}^{\infty} \Psi_{n, \wp}\left|\psi_{n} \zeta^{n-p}\right| \\
\leq & \sum_{n=p+1}^{\infty} \Psi_{n, \wp}\left|\psi_{n}\right|-|\mu-v| \\
& +\sum_{n=p+1}^{\infty} \Psi_{n, \wp} \nu\left|\psi_{n}\right| \\
= & \sum_{n=p+1}^{\infty} \Psi_{n, \wp}[1+\nu]\left|\psi_{n}\right|-|\mu-v| \leq 0
\end{aligned}
$$

\section{An Application}

In this section, we consider the suggested class $\Sigma_{\wp}^{\alpha}(\sigma,(1+\zeta) /$ $(1-\zeta))$ for all $\alpha \in[0,1]$. 
Theorem 21. Consider the class of analytic functions $\sum_{\wp}^{\alpha}(\sigma$, $(1+\zeta) /(1-\zeta))$. Then, the solution of the differential equation corresponds to this class is

$$
\left[Q^{m \alpha} \psi(\zeta)\right]=c_{1} \zeta^{\frac{(\sigma-1) \wp}{\sigma}}+\zeta^{\wp}\left(\frac{\left.2 \wp \zeta_{2} F_{1}(1,(\sigma+\wp) / \sigma, \wp / \sigma+2, \zeta)\right)+1}{(\sigma+\wp)}\right)
$$

where ${ }_{2} F_{1}(a, b, c ; \zeta)$ represents the hypergeometric function.

Proof. Suppose that $\psi \in \Sigma_{\wp}^{\alpha}(\sigma,(1+\zeta) /(1-\zeta))$. Then, it satisfies the differential equation

$$
\frac{(1-\sigma)}{\zeta^{\wp}}\left[Q^{m \alpha} \psi(\zeta)\right]+\left(\frac{\sigma}{\wp \zeta^{\wp-1}}\right)\left[Q^{m \alpha} \psi(\zeta)\right]^{\prime}=\frac{\omega(\zeta)+1}{1-\omega(\zeta)},
$$

where $\omega(0)=0$ and $|\omega|<1$. This leads to the solution

$$
\left[Q^{m \alpha} \psi(\zeta)\right]=\zeta^{((\sigma-1) \wp) / \sigma} \int_{0}^{\zeta}-\wp z^{\wp / \sigma-1}\left(\frac{\omega(z)+1}{\sigma(\omega(z)-1)}\right) d z
$$

To find the upper solution, we let $\omega(\zeta)=\zeta$. Thus, we have the differential equation

$$
\frac{(1-\sigma)}{\zeta^{\wp}}\left[Q^{m \alpha} \psi(\zeta)\right]+\left(\frac{\sigma}{\wp \zeta^{\wp-1}}\right)\left[Q^{m \alpha} \psi(\zeta)\right]^{\prime}=\frac{\zeta+1}{1-\zeta} .
$$

Rewrite the above equation as follows:

$$
\left[\mathscr{Q}^{m \alpha} \psi(\zeta)\right]^{\prime}+\frac{\wp(1-\sigma)}{\sigma \zeta}\left[Q^{m \alpha} \psi(\zeta)\right]=\left(\frac{\wp \zeta^{\wp-1}}{\sigma}\right)\left(\frac{1+\zeta}{1-\zeta}\right)
$$

Multiplying the above equation by the functional

$$
T(\zeta)=\exp \left(\int \frac{\wp(\sigma+\zeta-\sigma \zeta-1)}{\sigma \zeta(\zeta-1)} d \zeta\right)
$$

we obtain

$$
\begin{aligned}
& \zeta^{\wp(1 / \sigma-1)}\left[Q^{m \alpha} \psi(\zeta)\right]^{\prime}-\frac{\left[Q^{m \alpha} \psi(\zeta)\right]\left(\wp \zeta^{\wp(1 / \sigma-1)-1(\sigma+\zeta-\sigma \zeta-1)}\right)}{\sigma(1-\zeta)} \\
& =\left(\frac{\wp \zeta^{\wp / \sigma-1}}{\sigma}\right)\left(\frac{1+\zeta}{1-\zeta}\right) .
\end{aligned}
$$

Hence, it follows the solution (26).

Example 1. For

(i) $\wp=1, \sigma=0.5, c_{1}=0$, the solution is

$$
\left[Q^{m \alpha} \psi(\zeta)\right]=-\zeta-\frac{4(\zeta+\log (1-\zeta))}{\zeta}
$$

(ii) $\wp=1, \sigma=0.25, c_{1}=0$; the solution becomes

$$
\left[\mathbb{Q}^{m \alpha} \psi(\zeta)\right]=-\zeta-\frac{4\left(2 \zeta^{3}+3 \zeta^{2}+6 \zeta+6 \log (1-\zeta)\right)}{3 \zeta^{3}}
$$

(iii) $\wp=2, \sigma=0.5, c_{1}=0$; then the solution is given by the formula

$$
\left[Q^{m \alpha} \psi(\zeta)\right]=-\zeta^{2}-\frac{4\left(2 \zeta^{3}+3 \zeta^{2}+6 \zeta+6 \log (1-\zeta)\right)}{3 \zeta^{2}}
$$

\section{Conclusion}

Commencing overhead, we formulated a new parametric differential operator for a certain class of multivalently analytic functions. We investigated some geometric conducts of the operator connecting with the Janowski function, which is convex univalent in the open unit disk. As an application, we presented the formula of the suggested class involving the operator. For future works, one can generalize the suggested fractional operator using various classes of analytic functions such as meromorphic and harmonic functions.

\section{Data Availability}

No data were used to support this study.

\section{Conflicts of Interest}

The authors declare no conflict of interest.

\section{Authors' Contributions}

All authors contributed equally and significantly to writing this article. All authors read and agreed to the published version of the manuscript.

\section{References}

[1] S. Eker and S. Owa, "Certain classes of analytic functions involving Salagean operator," Journal of Inequalities in Pure and Applied Mathematics, vol. 10, no. 1, pp. 12-22, 2009.

[2] R. W. Ibrahim, "On generalized Srivastava-Owa fractional operators in the unit disk," Advances in Difference Equations, vol. 2011, no. 1, 10 pages, 2011.

[3] R. W. Ibrahim and J. M. Jahangiri, "Conformable differential operator generalizes the Briot-Bouquet differential equation in a complex domain," AIMS Mathematics, vol. 4, no. 6, pp. 1582-1595, 2019. 
[4] R. W. Ibrahim and D. Baleanu, "On a combination of fractional differential and integral operators associated with a class of normalized functions," AIMS Mathematics, vol. 6, no. 4, pp. 4211-4226, 2021.

[5] R. W. Ibrahim and D. Baleanu, "On quantum hybrid fractional conformable differential and integral operators in a complex domain," Revista de la Real Academia de Ciencias Exactas, Fisicas y Naturales. Serie A. Matemáticas, vol. 115, no. 1, pp. 1-13, 2021.

[6] B. Ahmad, M. G. Khan, M. K. Aouf, W. K. Mashwani, Z. Salleh, and H. Tang, "Applications of a new-difference operator in Janowski-type meromorphic convex functions," Journal of Function Spaces, vol. 2021, 10 pages, 2021.

[7] H.-Y. Zhang and H. Tang, "Fourth Toeplitz determinants for starlike functions defined by using the sine function," Journal of Function Spaces, vol. 2021, 7 pages, 2021.

[8] N. M. Alarifi and R. W. Ibrahim, "A new class of analytic normalized functions structured by a fractional differential operator," Journal of Function Spaces, vol. 2021, 9 pages, 2021.

[9] M. K. Aouf, A. O. Mostafa, and S. M. Madian, "Certain classes of meromorphic p-valent functions associated with MittagLeffler function," Proceedings of the Pakistan Academy of Sciences: A. Physical and Computational Sciences, vol. 58, no. 1, pp. 97-106, 2021.

[10] S. Ruscheweyh, Convolutions in Geometric Function Theory, Les Presses De L'Universite De Montreal, Montreal, 1982.

[11] P. L. Duren, "Univalent functions," in Grundlehren der Mathematischen Wissenschaften, vol. 259, Springer-Verlag, 1983, ISBN 0-387-90795-5.

[12] S. S. Miller and P. T. Mocanu, Differential Subordinations: Theory and Applications, CRC Press, 2000.

[13] T. N. Shanmugam, S. Sivasubramanian, and H. M. Srivastava, "Differential sandwich theorems for certain subclasses of analytic functions involving multiplier transformations," Integral Transforms and Special Functions, vol. 17, no. 12, pp. 889899, 2006.

[14] S. S. Miller and P. T. Mocanu, "Subordinants of differential superordinations," Complex Variables, vol. 48, no. 10, pp. 815-826, 2003.

[15] G. Dong and M.-S. Liu, "On certain subclass of Bazilevic functions," Journal of Inequalities in Pure and Applied Mathematics, vol. 8, no. 1, pp. 1-11, 2007.

[16] F. R. Keogh and E. P. Merkes, "A coefficient inequality for certain classes of analytic functions," Proceedings of American Mathematical Society, vol. 20, no. 1, pp. 8-12, 1969. 